\title{
Observation of Electromagnetically Induced Transparency in Numerical Magnetized Plasma Experiment
}

\author{
Eiichirou KAWAMORI \\ Institute of Space, Astrophysical and Plasma Sciences, National Cheng Kung University, Tainan, 70101, Taiwan
}

(Received 19 January 2012 / Accepted 1 May 2012)

\begin{abstract}
We report the observation, in a particle-in-cell simulation, of a phenomenon in which a magnetized plasma absorbing an electron cyclotron wave is rendered transparent by a pump wave, which is a classical analog to electromagnetically induced transparency (EIT) in quantum physics. Evident mode coupling between the probe electron cyclotron wave and the pump wave driving the longitudinal plasma oscillation, which is required for the realization of EIT, is identified in addition to an observation of the electron cyclotron wave recapturing propagation in the magnetized plasma.
\end{abstract}

(C) 2012 The Japan Society of Plasma Science and Nuclear Fusion Research

Keywords: electromagnetically induced transparency, electron cyclotron wave, mode coupling

DOI: $10.1585 /$ pfr.7.1301086

Conventional quantum electromagnetically induced transparency (EIT) is a phenomenon in which an optically thick medium is rendered transparent by controlled laser light [1]. A cold three-level atomic gas is typically employed as the medium. In EIT in three-level atomic systems, a probe light having the same frequency as an eigen frequency corresponding to the transition between two energy levels of the atom loses resonance with the atoms if another appropriately tuned control light (pump light) is injected. This transparency phenomenon is not a saturation or hole-burning phenomenon. Quantum EIT results from destructive interference (cancelation of dipole transitions) between eigen states of the atom and the lights, that is, a type of mode-coupling. EIT has many applications including the widely known light stopping [2], information transfer and lasing without inversion.

It is natural to consider expansion of this theoretical idea to classical oscillator systems such as plasma. EIT in plasma is a classical analog to quantum EIT in which plasma is assumed to be the medium. In the magnetized plasma EIT, the quantities equivalent to the eigen frequencies in three-level atomic EIT systems are considered to be the characteristic frequencies of a magnetized plasma, such as the plasma and cyclotron frequencies. EIT based on this idea was proposed for magnetized and unmagnetized plasma by Harris [3] and Litvak and Tokman [4], respectively. Although experimental studies of EIT in plasma were not conducted immediately when Harris first suggested the idea of plasma EIT in 1996 [3], the first experimental identification of EIT in magnetized plasma was recently reported [5].

This EIT in magnetized plasma creates a new branch at the electron cyclotron frequency when the frequency of

author'se-mail: kawamori@pssc.ncku.edu.tw the pump wave satisfies a matching condition $\omega_{\text {probe }} \approx$ $\omega_{\text {pe }}+\omega_{\text {pump }}$, where $\omega_{\text {probe }}, \omega_{\text {pe }}$ and $\omega_{\text {pump }}$ are the angular frequencies of the probe-wave, plasma wave and pump-wave, respectively. A plasma immersed in a background axial magnetic field absorbs the electromagnetic wave (probe wave), which is right-hand circularly polarized (RHCP) at the electron cyclotron frequency. Cold magnetized plasma is normally opaque to RHCP wave in the frequency band between $\omega=\omega_{\text {ce }}$ and $\omega=\omega_{\mathrm{RH}}$ (the stop band), where $\omega_{\mathrm{ce}}$ and $\omega_{\mathrm{RH}}$ are the electron cyclotron angular frequency and the right-hand cutoff angular frequency, respectively. If another appropriate electromagnetic wave (pump wave) is simultaneously injected, the electron cyclotron resonance (ECR) disappears, and the plasma becomes transparent to RHCP wave [6]. This disappearance of ECR is induced by cancelation of the polarization current of the ECR wave by mode coupling between the probe and pump waves $[4,6,7]$.

Plasma EIT has various potential applications including energy compression of electromagnetic microwaves [8], ion acceleration by the ponderomotive force, control of the spatial absorption profile of electron cyclotron heating waves in fusion plasmas [9] and free electron laser [10].

In this paper, we attempt to realize magnetized plasma EIT by a numerical simulation. The simulation code we employ is a full-relativistic particle-in-cell (PIC) code that is spatially one-dimensional and follows three components of the particle velocities. Therefore, the code enables us to examine plane-wave propagation in magnetized plasma. The parameters of the simulation are as follows: The time step $\Delta t$, grid size $\Delta z$, number of particles (ions and electrons) per grid $\Delta N$ and ratio between ion mass and electron mass $m_{\mathrm{i}} / m_{\mathrm{e}}$ are $0.1 \omega_{\text {ce }}^{-1}, c \Delta t, 10 \times 2$ and 1833.0, respectively, where $c$ is the speed of light in vacuum. Fig- 


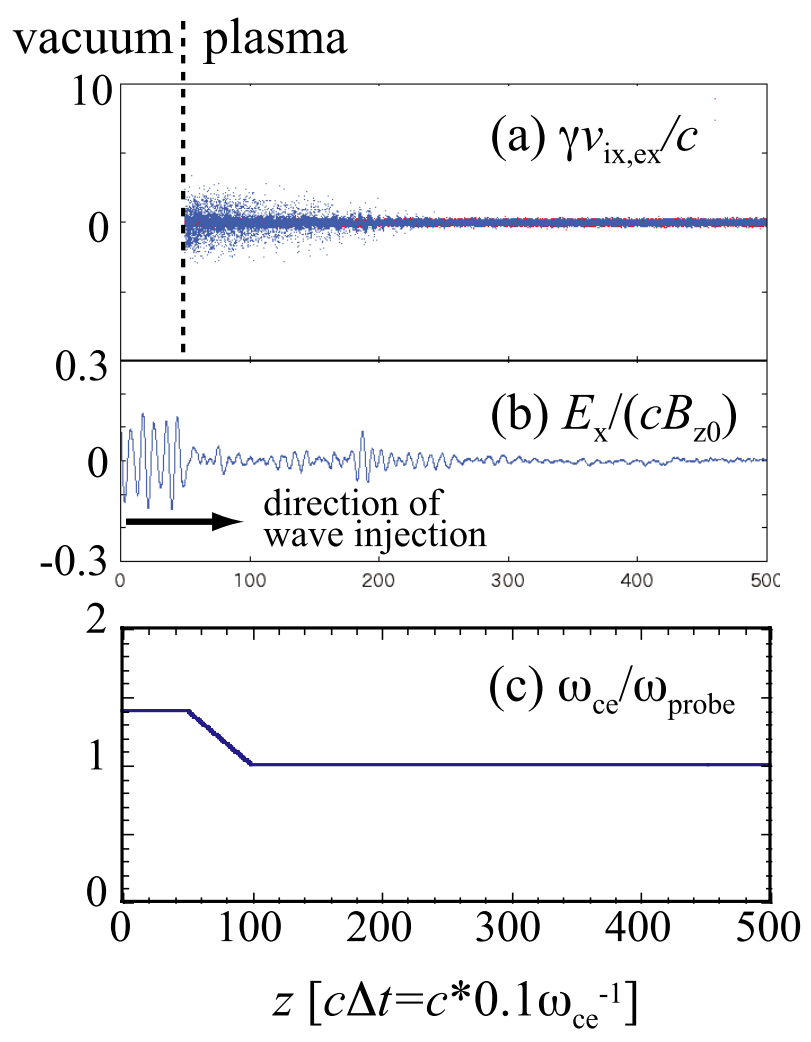

Fig. 1 Spatial (in $z$-direction) distributions of (a) ions and electrons with $x$ component of four-velocity $\gamma v_{x} / c$, where $\gamma \equiv\left(1-v^{2} / c^{2}\right)^{-1 / 2}$, (b) $x$ components of the normalized electric field $E_{x} /\left(c B_{z 0}\right)$, and (c) $\omega_{\text {ce }} / \omega_{\text {probe }}$, that is, the background magnetic field strength $B_{z 0}$.

ure 1 shows the configuration and a typical snapshot of the numerical experiment, specifically, the spatial distributions (in $z$ direction) of (a) the ions and electrons with an $x$ component of four-velocity $\gamma v_{x} / c$, where $\gamma \equiv(1-$ $\left.v^{2} / c^{2}\right)^{-1 / 2}$, (b) the $x$ components of the normalized electric field $E_{x} /\left(c B_{z 0}\right)$, and (c) $\omega_{\text {ce }} / \omega_{\text {probe }}$, that is, the normalized background magnetic field strength $B_{z 0}$. The plasma particles are confined by perfect reflection end-walls between $z=50 c \Delta t$ and $500 c \Delta t$. The leftmost region of the computational domain is a vacuum region from which the probe and pump waves are launched rightward. Both the rightgoing probe wave and a pump wave whose polarization is right-hand circular are injected from the vacuum region into a plasma along the background magnetic field (along the $z$-axis).

To achieve better impedance matching for the injected probe wave at the vacuum-plasma interface, the equilibrium magnetic field $\omega_{\text {ce }} / \omega_{\text {probe }}$ is set higher $(=1.4)$ at the vacuum-plasma interface $(z=50 c \Delta t)$ and decreases to 1.0 at $z=100 c \Delta t$. Although $\operatorname{div} \boldsymbol{B}_{0}=0\left(\boldsymbol{B}_{0}\right.$ : the equilibrium magnetic field) is not satisfied in the region where $\omega_{\text {ce }} / \omega_{\text {probe }}$ is not constant $(z=50-100 c \Delta t)$, the wave fields are properly treated in the entire region. That is, $\operatorname{div} \boldsymbol{B}=0$ for the wave magnetic field is guaranteed in the entire re-

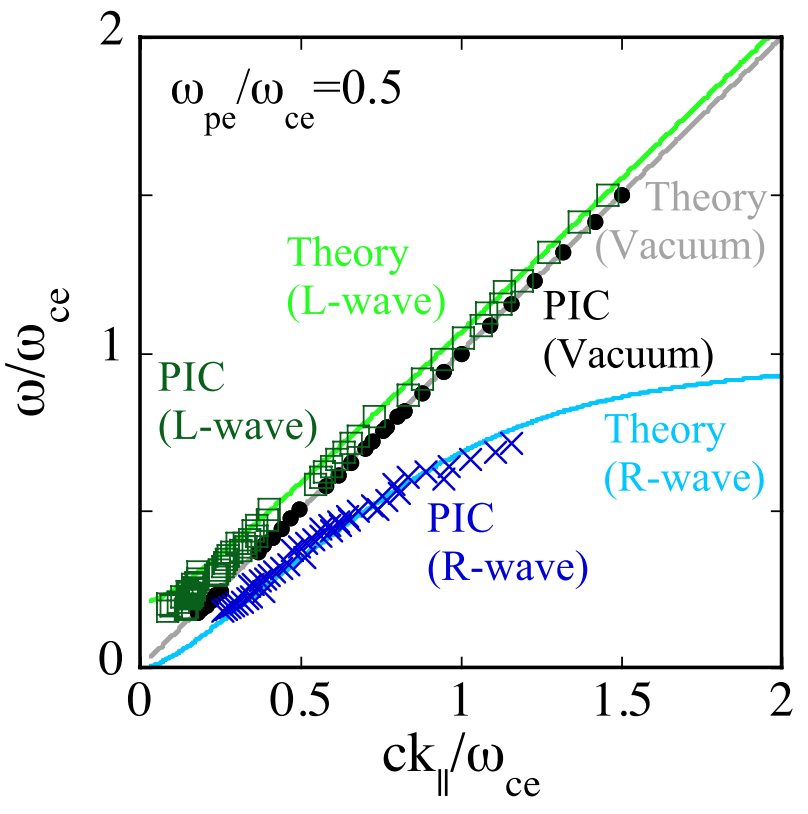

Fig. 2 Dispersion relation of waves propagating parallel to background magnetic field obtained from the PIC code simulation (crosses, open squares and closed circles) and theory of the CPDR.

gion. The only effect of $\operatorname{div} \boldsymbol{B}_{0} \neq 0$ is that the mirror force is neglected for the particles in the region $(z=50-100 c \Delta t)$. This neglect of the mirror force makes it possible to prepare a uniform equilibrium electron (and ion) density along the $z$-axis.

First, we checked the validity of the PIC code by comparing the dispersion relation obtained from the PIC simulation with the cold plasma dispersion relation (CPDR). For this test, we launched only a probe wave into a magnetized plasma without pump-wave injection. Here, $\omega_{\mathrm{pe}} / \omega_{\mathrm{ce} 0}$ is 0.5 , where $\omega_{\text {ce } 0}$ is the electron cyclotron frequency in the flat magnetic field region $(z=100-500 c \Delta t)$. Figure 2 shows the dispersion relation $\left(\omega / \omega_{\text {ce }}\right.$ vs. $\left.c k_{\|} / \omega_{\text {ce }}\right)$ in the electron cyclotron frequency band. Blue crosses and green squares represent the PIC simulation results in the magnetized plasma for right-hand and left-hand polarized waves, respectively. Closed black circles represent wave propagation in the vacuum. Solid curves are calculated from CPDR. The PIC results agree well with the theoretical curves obtained by CPDR. By another simulation, we confirmed that the PIC code accurately demonstrates an electrostatic mode, that is, the longitudinal plasma oscillation, although the result is not shown in this paper.

The following three wave-injection methods were applied in an attempt to identify EIT by switching the probe wave or pump wave on or off: (i) probe-wave injection only, (ii) pump-wave injection only and (iii) injection of both probe and pump waves. The amplitudes of the probe wave and pump wave $|E| /\left(c B_{z 0}\right)$ were 0.03 and 0.1 , respectively. The angular frequencies of the probe 


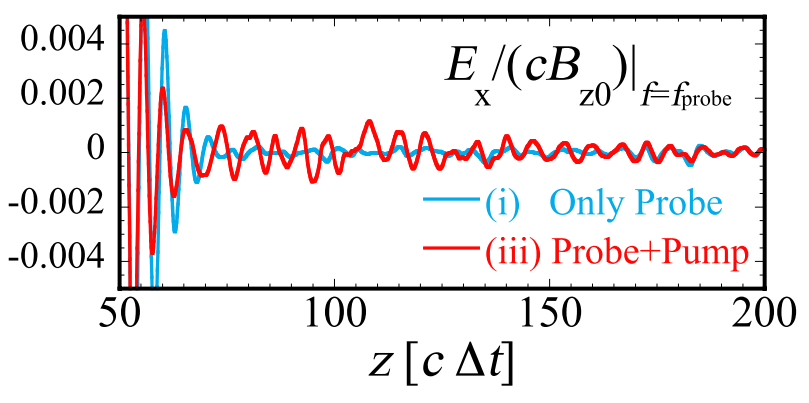

Fig. 3 Spatial profiles of $E_{x}\left(E_{\perp}\right)$ of probe wave for probe-wave injection only (light blue) and injection of both probe and pump waves (red). These are obtained from the bandpass-filtered wave amplitude using a FIR filter having a frequency range of $0.99 \omega_{\mathrm{ce}}-1.01 \omega_{\mathrm{ce}}$.

wave $\omega_{\text {probe }}$ and pump wave $\omega_{\text {pump }}$ were $\omega_{\mathrm{ce} 0}$ and $0.7 \omega_{\mathrm{ce} 0}$, respectively. The plasma angular frequency $\omega_{\text {pe }}$ at the given plasma density satisfies the EIT matching condition $\omega_{\text {probe }} \approx \omega_{\text {pe }}+\omega_{\text {pump }}$.

Figure 3 shows snapshots of spatial profiles of the normalized probe wave electric field $E_{x} /\left(c B_{z 0}\right)$ for (i) probewave injection only and (iii) injection of both probe and pump waves. A finite impulse response (FIR) filter extracts $E_{x} /\left(c B_{z 0}\right)$ whose angular frequency is $\omega_{\text {ce } 0}$ from the full-band data. The electron cyclotron wave recaptures its propagation when accompanied by the pump wave (case (iii)).

Figure 4 shows Fourier power spectra of the electric fields $E_{x}\left(E_{\perp}\right)$ (top) and $E_{z}\left(E_{\|}\right)$(bottom) measured at $z=110.0 c \Delta t$, where the axial magnetic field corresponds to the ECR field. The window size of the discrete Fourier transformation is 4096, and the number of ensembles used to calculate the power spectrum is 4 . As shown by the light blue curve in Fig. 4 (top), the spectrum of $E_{\perp}$ in case (i), only the probe-wave injection, has no peak at $f=\omega_{\text {probe }} /(2 \pi) \sim 0.16$ because the probe wave is resonantly absorbed by the electrons. On the other hand, in the case (iii), the pump-wave injection and probe-wave injections (red curve), two peaks in $E_{\perp}$ are clearly observed at $f \sim 0.11$ and $f \sim 0.16$ which correspond to $\omega_{\text {pump }} /(2 \pi)$ and $\omega_{\text {probe }} /(2 \pi)$, respectively.

When the pump wave is injected in addition to the probe wave, longitudinal plasma oscillation is excited as shown by the red curve in Fig. 4 (bottom). The frequency of this longitudinal oscillation agrees well with $\omega_{\text {pe }} /(2 \pi)$. These three frequencies, $f \sim 0.059,0.107$, and 0.163 , observed in case (iii) accurately satisfy the EIT matching condition $\omega_{\text {probe }} \approx \omega_{\text {pe }}+\omega_{\text {pump. }}$.

Figure 5 shows the phase difference $\Delta \phi$ between $E_{x}$ and $E_{y}$ in case (iii), the injection of both probe and pump waves. At $f \sim 0.16, \Delta \phi$ is $-\pi / 2$, indicating that the electric field of the wave with $f=\omega_{\text {probe }} /(2 \pi)$ is right-hand polarized.

We examine the possibility of stimulated Raman scat-
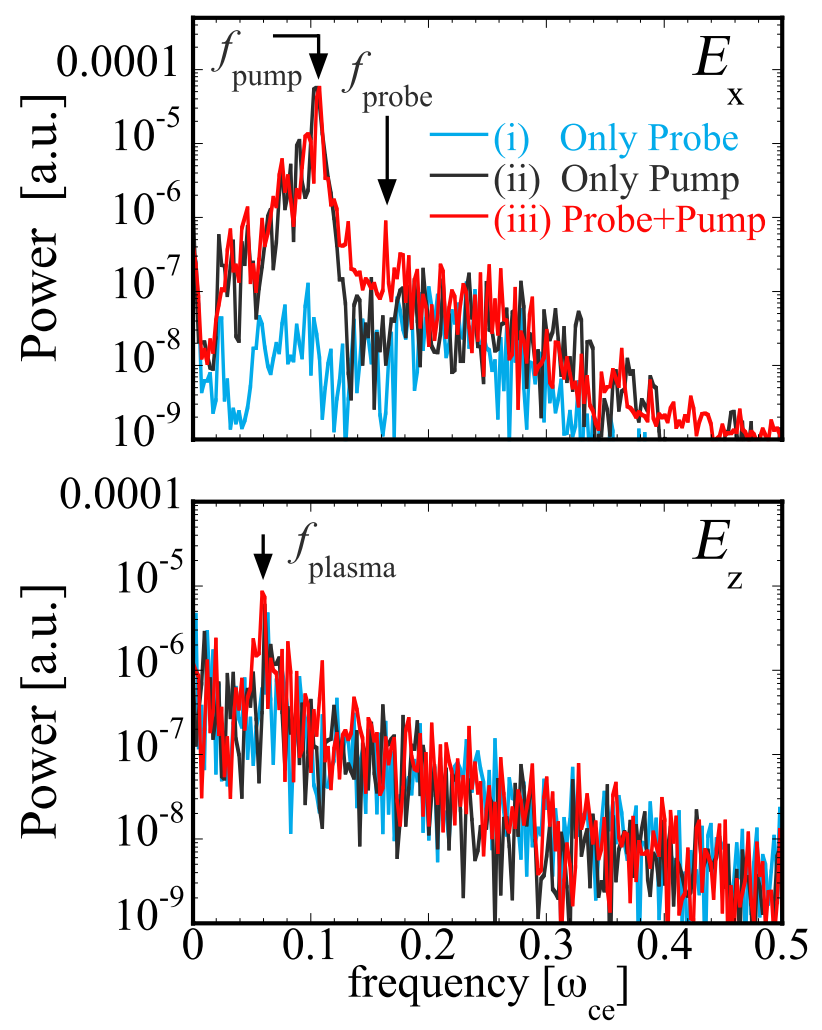

Fig. 4 Fourier power spectra of $E_{x}\left(E_{\perp}\right)$ and $E_{z}\left(E_{\|}\right)$measured at $z=110.0$. Curves show probe-wave injection only (light blue), pump-wave injection only (black), and injection of both probe and pump waves (red).

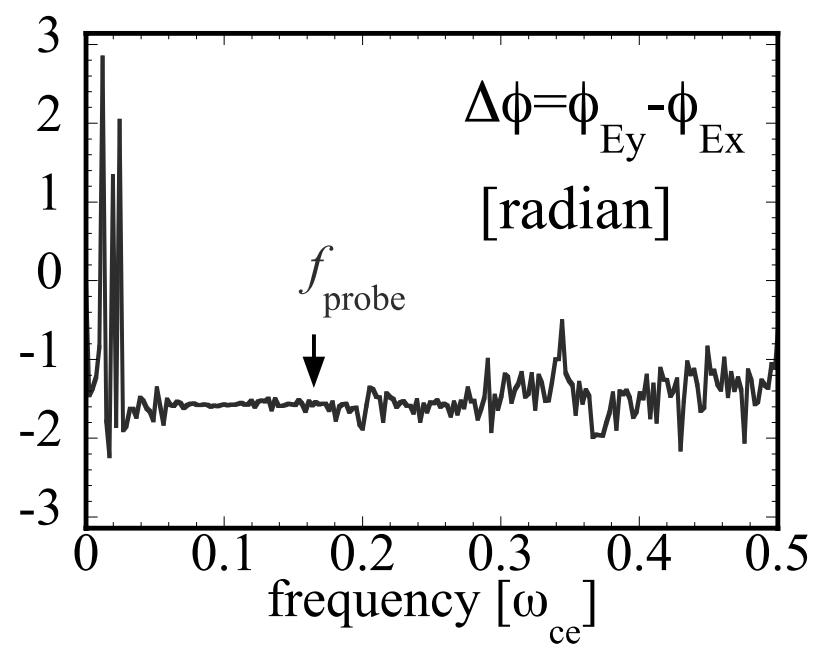

Fig. 5 Phase difference $\Delta \phi$ between $E_{x}$ and $E_{y}$ for injection of both probe and pump waves.

tering (SRS) of the pump wave. In case (ii), shown by the gray curve in Fig. 4 (top), the peak at $f=\omega_{\text {probe }} /(2 \pi) \sim$ 0.16 is absent, whereas a peak produced by the pump wave appears. This result rules out the occurrence of SRS of the pump wave by the plasma wave to the electron cyclotron wave in this simulation. 
Relativistic effects make almost no contribution to EIT creation in the simulation. The average relativistic factor $\gamma$ of the electrons is small $(\sim 1.05)$ in the EIT demonstration.

These results indicate that pump-wave injection renders the plasma transparent to the electron cyclotron probe wave as a result of coupling between the probe and pump waves which drives the plasma oscillation.

In summary, we have shown evidence of EIT in magnetized plasma in a PIC simulation. Plasma becomes transparent to the electron cyclotron wave when a tuned pump wave is injected. Spectral analysis of the measured wave electric field indicates the mode coupling between the probe and pump waves that excites longitudinal plasma oscillation. This is consistent with the theory reported elsewhere $[4,6]$.

\section{Acknowledgments}

This work was partly supported by Grants-in-Aid 97-
2112-M-006-016-MY3 from the National Science Council of Taiwan.

[1] K.J. Boller, A. Imamoglu and S.E. Harris, Phys. Rev. Lett. 66, 2593 (1991).

[2] L.V. Hau, S.E. Harris, Z. Dutton and C.H. Behroozi, Nature 397, 594 (1999).

[3] S.E. Harris, Phys. Rev. Lett. 77, 5357 (1996).

[4] A.G. Litvak and M.D. Tokman, Phys. Rev. Lett. 88, 095003 (2002).

[5] E. Kawamori, W.-J. Syugu, S.-X. Song, T.-Y. Hsieh and C.Z. Cheng, Phys. Rev. Lett. 108, 075003 (2012).

[6] G. Shvets and J.S. Wurtele, Phys. Rev. Lett. 89, 115003 (2002).

[7] E. Kawamori, Phys. Plasmas 17, 102108 (2010).

[8] M. Tushentsov et al., IEEE Trans. Plasma Science 33, 23 (2005).

[9] E. Kawamori, Plasma Phys. Control. Fusion 53, 085015 (2011).

[10] A.B. Matsko and Y.V. Rostovtsev, Phys. Rev. E 58, 7846 (1998). 